University of Wollongong

Research Online

Faculty of Arts, Social Sciences and Humanities

- Papers

Faculty of Arts, Social Sciences \& Humanities

2020

A systematic review of interventions to improve the dietary intake, physical activity and weight status of children attending family day care services

Sze Yoong

Melanie Lum

Jannah Jones

Erin Kerr

University of Wollongong, emk833@uowmail.edu.au

Maryann Falkiner

See next page for additional authors

Follow this and additional works at: https://ro.uow.edu.au/asshpapers

Research Online is the open access institutional repository for the University of Wollongong. For further information contact the UOW Library: research-pubs@uow.edu.au 


\title{
A systematic review of interventions to improve the dietary intake, physical activity and weight status of children attending family day care services
}

\author{
Abstract \\ Objective:To (i) identify and synthesise findings from interventions to improve the dietary intake, physical \\ activity and weight status of children aged 0-6 years attending family day care services; and (ii) assess \\ the impact of interventions on family day care environments, intervention cost and adverse \\ outcomes.Design:Medline in Process, PsycINFO, ERIC, Embase, CINAHL, CENTRAL and Scopus \\ databases were searched in March 2019. Studies were included if they (i) evaluated an intervention to \\ improve the diet, physical activity and/or weight of children aged 0-6 years; (ii) were delivered in family \\ day care services; (iii) targeted child diet, physical activity and/or weight; and (iv) used a parallel control \\ group design. Screening was undertaken by two reviewers with disagreements resolved by a third \\ reviewer.Setting:Family day care services, also known as family childcare homes.Participants:Children \\ aged 0-6 years attending family day care services. Results:In total, 8977 titles were retrieved, and 199 full- \\ texts reviewed. No studies met the inclusion criteria for the primary outcome; however, two studies \\ reporting on the secondary outcome of family day care environments were included. The 4-year \\ community-wide obesity prevention programme and the 12-month train-the-trainer programme both \\ reported statistically significant improvements in the healthy eating and physical activity environments of \\ family day care, compared to cross-sectional state-average control groups.Conclusions:Findings highlight \\ few existing interventions in family day care services and a need for high-quality controlled trials to \\ identify effective interventions to improve children's diet, activity and weight in this setting.

\section{Publication Details} \\ Yoong, S., Lum, M., Jones, J., Kerr, E., Falkiner, M., Delaney, T., McCrabb, S., Chai, L., Seward, K. \& Grady, A. \\ (2020). A systematic review of interventions to improve the dietary intake, physical activity and weight \\ status of children attending family day care services. Public Health Nutrition, 23 (12), 2211-2220.
}

\section{Authors}

Sze Yoong, Melanie Lum, Jannah Jones, Erin Kerr, Maryann Falkiner, Tessa Delaney, Sam McCrabb, Li Chai, Kirsty Seward, and Alice Grady 


\title{
A systematic review of interventions to improve the dietary intake, physical activity and weight status of children attending family day care services
}

\author{
Sze Lin Yoong ${ }^{1,2,3,4, *}$, Melanie Lum ${ }^{1,2,3}{ }^{0}$, Jannah Jones ${ }^{1,2,3,4}$, Erin Kerr ${ }^{5}$, \\ Maryann Falkiner ${ }^{2}$, Tessa Delaney 1,2,3,4 Sam McCrabb', Li Kheng Chai ${ }^{1,2,3}$, \\ Kirsty Seward ${ }^{1}$ and Alice Grady 1,2,3,4 (1) \\ 'University of Newcastle, School of Medicine and Public Health, Callaghan, NSW 2308, Australia: ${ }^{2}$ Hunter New \\ England Local Health District, Population Health, Wallsend, NSW 2287, Australia: ${ }^{3}$ Hunter Medical Research \\ Institute, University of Newcastle, New Lambton Heights, NSW 2305, Australia: ${ }^{4}$ Priority Research Centre for Health \\ Behaviour, University of Newcastle, Callaghan, NSW 2308, Australia: ${ }^{5}$ Early Start and School of Education, \\ University of Wollongong, Wollongong, NSW 2522, Australia
}

Submitted 10 July 2019: Final revision received 11 November 2019: Accepted 20 December 2019: First published online 8 May 2020

\begin{abstract}
Objective: To (i) identify and synthesise findings from interventions to improve the dietary intake, physical activity and weight status of children aged 0-6 years attending family day care services; and (ii) assess the impact of interventions on family day care environments, intervention cost and adverse outcomes.

Design: Medline in Process, PsycINFO, ERIC, Embase, CINAHL, CENTRAL and Scopus databases were searched in March 2019. Studies were included if they (i) evaluated an intervention to improve the diet, physical activity and/or weight of children aged 0-6 years; (ii) were delivered in family day care services; (iii) targeted child diet, physical activity and/or weight; and (iv) used a parallel control group design. Screening was undertaken by two reviewers with disagreements resolved by a third reviewer.

Setting: Family day care services, also known as family childcare homes.

Participants: Children aged 0-6 years attending family day care services.

Results: In total, 8977 titles were retrieved, and 199 full-texts reviewed. No studies met the inclusion criteria for the primary outcome; however, two studies reporting on the secondary outcome of family day care environments were included. The 4-year community-wide obesity prevention programme and the 12 -month trainthe-trainer programme both reported statistically significant improvements in the healthy eating and physical activity environments of family day care, compared to cross-sectional state-average control groups.

Conclusions: Findings highlight few existing interventions in family day care services and a need for high-quality controlled trials to identify effective interventions to improve children's diet, activity and weight in this setting.
\end{abstract}

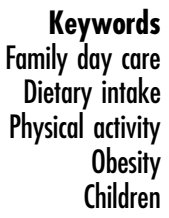

Globally, the prevalence of overweight and obesity in young children is high, with 41 million children under the age of 5 reported to be overweight or obese in $2016^{(1)}$. Obesity in childhood is associated with both short- and long-term health conditions ${ }^{(1-3)}$. Studies report that excess weight tracks into adulthood, increasing the lifetime risk of a range of diseases ${ }^{(2,4)}$. The primary determinants of excessive weight gain are poor diet and physical inactivity (including excessive sedentary behaviour) ${ }^{(5)}$. While international guidelines for physical activity, sedentary behaviour and healthy eating in young children exist, research suggests that the majority of children do not meet such recommendations ${ }^{(6,7)}$. As such, leading organisations, including the World Health Organization (WHO), recommend that these risk factors be targeted in community-based interventions to achieve the healthy weight status of children ${ }^{(8)}$.

Early childhood education and care services represent an important setting for implementing health-promoting interventions, given that they provide access to a significant proportion of young children for prolonged periods 
throughout the day ${ }^{(9)}$. In Australia, such services primarily consist of centre-based childcare services and family day care services $^{(10)}$, also known as family childcare homes and childminding in the United Kingdom. Centre-based childcare services are typically run in a purpose-built facility, have set hours of operation and can accommodate a larger number of children ${ }^{(11)}$. Family day care services, which usually provide care to a smaller number of children within an individual provider's own home, where hours of operation and regulatory structures vary, is the focus of the current review ${ }^{(11)}$.

Family day care services are the third largest provider of care for children in Australia who do not attend school (approximately $10 \%$ of the population) ${ }^{(10)}$, and almost one million ( $11 \%$ ) children in the United States access such care $^{(12,13)}$. Family day care services may also be accessed by more disadvantaged groups due to their overall lower daily fees $^{(9)}$, with the proportion of Australian families accessing such services increasing as income reduces. In the United States, a study in family day care services reported that $63 \%$ of children were of African American background who report poorer health outcomes ${ }^{(14)}$.

There is a significant opportunity to improve children's obesogenic behaviours while attending family day care services. A systematic review examining preschoolers' physical activity levels in family day care services has found that the average time preschoolers spent in moderate- to vigorous-intensity activity and total physical activity was 5.8 and $10.4 \mathrm{~min} / \mathrm{h}$, respectively ${ }^{(15)}$, which is lower than the recommendation of $120 \mathrm{~min}$ of activity in care ${ }^{(16)}$. A review of screen-viewing has found that children attending family day care services engaged in 108-114 min of screen time per day compared to 6-78 min for children attending centre-based care ${ }^{(17)}$. While no review of dietary intake exists, a number of cross-sectional studies of children attending family day care services in the United States have reported that diet quality scores varied from 59 to 64 out of a possible 100, highlighting significant opportunities for improvement ${ }^{(14,18)}$.

The majority of research to date has focused on improving child's diet, activity and weight in centre-based care, such as preschools and long-day-care centres. The interventions undertaken in centre-based care include those targeting environmental enhancements (e.g. interventions that aim to change the availability of food and/or play equipment), curriculum (e.g. interventions that schedule time for structured play or healthy eating opportunities), policy (e.g. interventions that demonstrate organisational commitment) and education (e.g. interventions that seek to improve the skills and knowledge of staff, children and parents) ${ }^{(19-21)}$. While family day care services can act in accordance with regulations and standards similar to centre-based childcare services ${ }^{(11)}$, the ability of providers to deliver health promotion programmes is likely to differ from centre-based childcare ${ }^{(15)}$. The organisational nature of family day care services, which involves smaller numbers of children of wider age ranges, single caregiver environments, as well as different physical infrastructures and levels of staff training, is likely to present unique challenges with the implementation of health promotion programmes ${ }^{(22)}$. Previous studies have also highlighted that family day care providers may have poorer health behaviours, which may impact their ability to become positive role models for children in their care ${ }^{(23)}$.

A recent review by Francis et $a l^{(15)}$ in 2018 sought to describe US-based studies that examined the nutrition and physical activity-promoting environments of family day care services. This review of observational studies has found that there are significant opportunities to improve the physical, policy and sociocultural environments of US-based family day care services to promote healthy eating and physical activity among children attending these services. In particular, the review highlighted that a lack of comprehensive written policies, lack of training for family day care providers, inaccurate nutrition beliefs, poor communication with families, lack of equipment and space for play, and poor feeding practices may need to be targeted to improve child's diet, activity and weight-related behaviour. To our knowledge however, there has been no previous synthesis of the impact of intervention studies that seek to improve the diet, physical activity and weight status of young children attending family day care services. Such a review is warranted to identify effective approaches and opportunities to enhance future research in this setting. As such, this systematic review sought to identify and assess the effectiveness of interventions to improve the dietary intake, physical activity and weight status of children aged 0-6 years attending family day care services. Secondary aims of the review were to examine the impact of the interventions on family day care services' health-promoting environments, policies or practices, similar to that defined by Francis et al. ${ }^{(15)}$ Additionally, adverse outcomes and costs of the intervention were also examined. Lastly, the review sought to describe ongoing studies that met the inclusion criteria.

\section{Methods}

\section{Registration}

This review employed rigorous review procedures as recommended by the Cochrane Collaboration ${ }^{(24)}$, including pre-specifying review questions and screening by two individuals at every stage. The review was prospectively registered with the International Prospective Register of Systematic Reviews (CRD42017077078) and is reported in accordance with the PRISMA guidelines ${ }^{(25)}$.

\section{Information sources and search strategy}

A computer-based literature search was carried out in March 2019. The search strategy was developed in consultation with an experienced medical research librarian 
and conducted on the following electronic databases: Medline in Process, PsycINFO, ERIC, Embase, CINAHL, CENTRAL and Scopus. The search strategy included search terms for the setting (childcare services and family day care services) as well as 'dietary intake/nutrition', 'physical activity' and 'weight'-related search terms used in a Cochrane systematic review ${ }^{(21)}$ undertaken by the authors (see online Appendix A for search strategy). The reference lists of all potentially relevant studies and systematic reviews were also searched by one author (M.L.).

\section{Eligibility criteria}

\section{Inclusion criteria}

To be included in this review, studies must have examined an intervention aiming to improve the diet, physical activity and/or weight status of children attending family day care services, with a parallel control arm, including randomised, cluster-randomised, factorial trial, interrupted time series, multiple baseline, stepped wedge and any controlled nonrandomised trial. For the purpose of this review, the term 'family day care services' refers to a formal type of childcare service where providers deliver care to a small group of children typically in the provider's own home. Family day care services are an approved form of childcare, generally cater to children prior to compulsory schooling and are usually required to undergo licensing and accreditation processes ${ }^{(11)}$.

All interventions that promote healthy eating, nutrition and/or physical activity, reduce sedentary behaviour or prevent unhealthy weight gain were eligible for inclusion. Interventions could be environmental, organisational or policy- and practice-related; singular or multi-component; and delivered by research staff, family day care service staff or any other organisation or expert. These could include interventions targeting environmental enhancements, curriculum, policy and staff, as well as parent and children education and knowledge. Interventions that target factors influencing the operation of family day care services in relation to professional guidelines, accreditation standards, food procurement strategies or other interventions were also eligible for inclusion. There were no restrictions on intervention duration. Interventions that targeted family day care services as part of a broader multi-component intervention targeting child's dietary intake and physical activity were eligible for inclusion if outcomes from the family day care services could be isolated.

Outcomes included any objective or subjective measures of dietary intake, physical activity or sedentary behaviour and measures of weight status. Secondary outcomes included those related to family day care services' health-promoting environments, policies or practices, adverse outcomes or any estimate of intervention costs. Examples of family day care environments include changes to the physical environment (availability of healthier foods, water, play equipment), policy (the presence and content of healthy eating and physical activity guidelines) and sociocultural environments (role modelling of healthy eating behaviours by family day care providers) ${ }^{(15)}$ of the family day care service. This may be assessed via audits of service records, questionnaires or surveys of staff, service managers, other personnel or parents; direct observation or recordings; examination of routine information collected from government departments or other sources.

\section{Exclusion criteria}

Manuscripts or reports not published in English were excluded, as were studies involving centre-based childcare services only (e.g. preschools, long-day-care services, kindergartens), as well as informal types of childcare provided in the child's own home (e.g. care given by grandparents, nannies, au pairs, babysitters). Studies that did not have an intervention component delivered within family day care services were also excluded (i.e. studies that recruited participants from the setting only). Interventions that focused specifically on examining malnutrition/malnourishment were excluded, as were those examining obesity treatment (i.e. those included only overweight/obese children).

\section{Study selection}

Screening procedures developed for the review were pilot-tested before use and undertaken using an online systematic review tool, Covidence ${ }^{(26)}$. Two authors (among S.L.Y, M.L., L.K.C., S.M.) independently screened all titles and abstracts. Full-text manuscripts of all potentially relevant studies were obtained and independently assessed for eligibility by two authors (among M.L., L.K.C., M.F., K.S., E.K., S.L.Y.). In instances where conflicts regarding the eligibility of studies were not resolved via consensus, a decision was made by a third author (among S.L.Y., L.K.C., A.G.). Authors of potentially relevant studies were contacted to request provision of additional data. Authors of published protocols were also approached to request further details of any publications in the press.

\section{Data collection processes}

Two authors (A.G. and M.L.) independently extracted information using a data extraction form developed based on the recommendation included in the Cochrane Public Health Group Guide for Developing a Cochrane Protocol ${ }^{(27)}$. Data extracted included: (1) study information, including study design, date of publication, childcare service type (e.g. family day care service), country, recruitment rate, service/participants' demographic/socioeconomic characteristics and number of experimental conditions; (2) characteristics of the intervention, including the duration, number of contacts, intervention components and implementation strategies classified according to the Effective Practice and Organisation of Care (EPOC) taxonomy ${ }^{(28)}$, theoretical underpinning and delivery modalities, as well as a description of control; (3) primary and secondary outcomes, including data collection methods, validity of measures used, effect 
size and measures of outcome variability, where possible; (4) intervention costs and adverse outcomes; (5) source(s) of research funding and potential conflicts of interest. All discrepancies in the data extraction process were resolved between review authors by consensus.

\section{'Risk of bias' assessment}

Two authors (A.G. and M.L.) independently assessed the risk of bias using the 'Risk of Bias' tool described in the Cochrane Handbook for Systematic Reviews of Interventions $^{(24)}$. Each included study was given a 'risk of bias' assessment ('high', 'low' or 'unclear') based on the consideration of methodological characteristics (random sequence generation, allocation concealment, blinding of participants and personnel, blinding of outcome assessment, incomplete outcome data, selective outcome reporting and 'other' potential sources of bias). Discrepancies were resolved between authors by consensus. We included 'potential confounding' as an additional criterion for the assessment of risk of bias due to the inclusion of non-
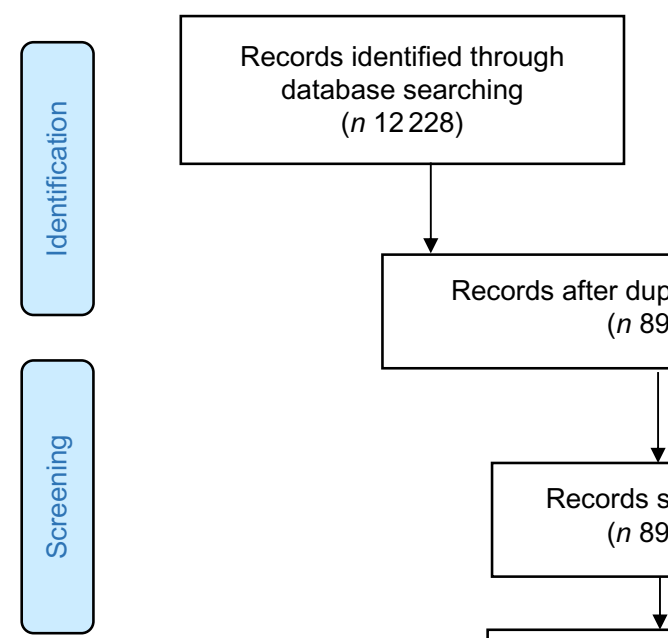

\section{Analysis}

We were unable to undertake a meta-analyses as the included studies were highly heterogeneous. Instead, we narratively described the intervention and outcomes as reported by each study.

\section{Results}

\section{Study selection}

The electronic search yielded 8965 citations. An additional twelve records were identified from checking the reference lists of potentially relevant systematic reviews, publications and study protocols. Following the screening of titles and abstracts, 199 full texts were obtained for further review. Two controlled studies that assessed the impact of interventions on family day care service's health-promoting environments were included; however, both did not measure any impact on child-level outcome (primary aim). The primary reasons for excluding studies from the review are presented in Fig. 1. One study was excluded as it did not address diet, physical activity and sedentary behaviour;

Additional records identified through other sources

(n 12)

Records after duplicates removed (n 8977)
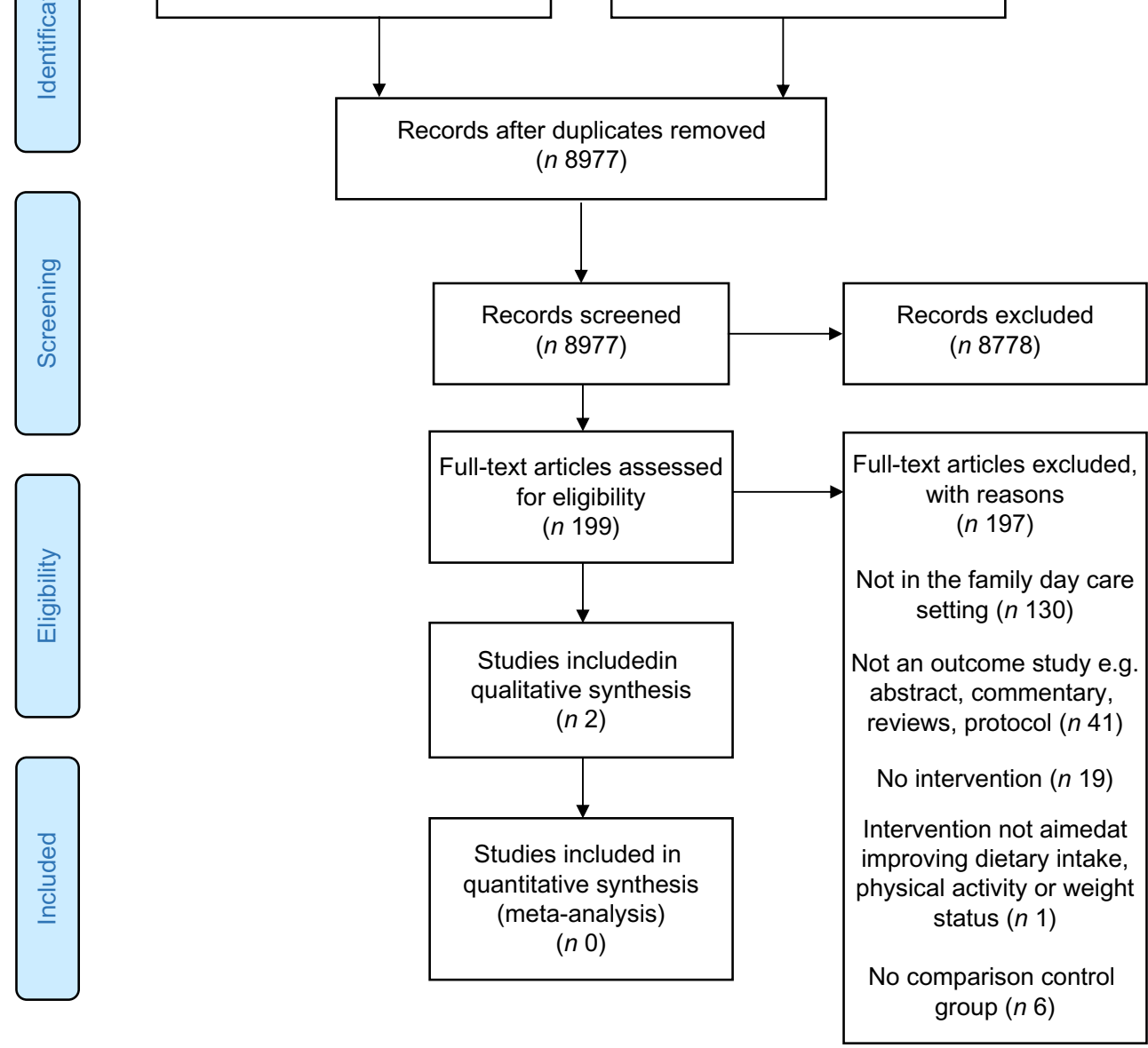

Fig. 1 (colour online) Study flow diagram 
rather, it examined an intervention to improve children's social and emotional wellbeing. Although studies may have examined interventions to improve family day care environments, not having a parallel control group resulted in their exclusion. Online Appendix B provides an overview of such types of previous interventions undertaken in this setting ${ }^{(29-36)}$.

\section{Study characteristics}

The trials were conducted in the United States $(n 1)^{(37)}$ and Australia $\left(n\right.$ 1) ${ }^{(30)}$, and both published in 2011 (conducted 2004-9). Participants were family day care schemes (the managing organisational structure) and family day care providers (individuals providing care to children). Eighteen family day care schemes participated in one trial ${ }^{(30)}$, with family day care providers ranging from 251 to 533 across both trials. Both studies were of a quasiexperimental design, comparing the intervention participants to a cross-sectional sample drawn from the same state in which the interventions took place. Characteristics of included studies are reported in Table 1.

\section{Risk of bias}

Both studies were judged as having a high risk of selection bias (i.e. random sequence generation and allocation concealment) as they were non-randomised trials (see Table 2). Further, for both trials, there was insufficient information to assess for the following criteria: selective outcome reporting, contamination and potential confounding. The study by Trost et al. ${ }^{(37)}$ was judged as having a low risk for incomplete outcome data and baseline imbalance.

\section{Interventions}

Intervention duration ranged between 12 months ${ }^{(37)}$ and 4 years $^{(30)}$ between the two studies. One study aimed to determine the effectiveness of a community-wide programme in reducing obesity and promoting healthy eating and active play among young children (aged $0-5$ years) ${ }^{(30)}$; the other aimed to assess the impact of a train-the-trainer intervention on family day care services' healthy eating and physical activity policies and practices ${ }^{(37)}$. The study by de Silva-Sanigorski et al. $^{(30)}$ used health promotion principles by Nutbeam ${ }^{(38,39)}$ and the socioecological framework $^{(40,41)}$ to guide intervention development, while the other study did not report the use of theory ${ }^{(37)}$. Both studies used a range of strategies delivered to family day care providers, including educational meetings (training), educational outreach visits and educational materials.

\section{Measures}

Both studies used self-reported environmental surveys to assess the impact of the interventions. de Silva-Sanigorski et $a l .{ }^{(30)}$ used an environmental audit to capture factors in the physical, policy, sociocultural and economic environment of family day care providers, whereas Trost et $a l^{(37)}$ employed the validated Nutrition and Physical Activity Self-Assessment for Child Care self-assessment instrument ${ }^{(42,43)}$ to capture the implementation of nutrition and physical activity policies and practices within family day care.

\section{Outcomes}

One study reported statistically significant improvements in the intervention group on a number of environmental outcomes, including increased active play opportunities and more healthy eating rules and supportive meal time practices compared to the control group ${ }^{(30)}$. The other study reported statistically significant improvements in healthy eating and physical activity environment scores among the intervention group compared to the control ${ }^{(37)}$. Neither trial measured the impact on child outcomes, nor reported intervention costs or adverse effects.

\section{Ongoing studies}

Two ongoing studies were also identified. The first was a two-arm, cluster randomised controlled trial involving 150 family day care providers and 450 children. The 9-month intervention conducted in the United States targeted the health, knowledge and skills of the family day care provider to create environments to encourage healthy eating and physical activity in children, and adopt sound business practices ${ }^{(44)}$. The second protocol was a cluster-randomised trial with 132 family day care providers and 396 children in the United States. The study compared the intervention aimed at improving food and physical activity environments with an active comparison group that provided resources related to literacy and school readiness $^{(45)}$

\section{Discussion}

This review is the first to synthesise findings from controlled trials, conducted in family day care services, that examined interventions to improve child's diet, physical activity and weight status. We could not identify any studies that met the pre-specified inclusion criteria for the primary outcomes; however, two studies that examined the secondary outcomes of family day care service's healthy eating and/or physical activity environments were included. Additionally, two ongoing studies conducted in the United States were identified, which are likely to contribute considerably to the evidence base once findings are disseminated ${ }^{(44,45)}$. Given the reach of family day care services, access to potentially vulnerable groups and the low levels of activity and poor diets of children attending such settings ${ }^{(12,22)}$, findings from this review clearly indicate a need for future controlled trials to identify effective obesity prevention interventions in this setting. 


\section{Neselic Health Nutrition}

Table 1 Study characteristics of included trials

First author, year published, country

Methods

Participants

Intervention

Outcomes

Conflicts of interest

de Silva-Sanigorski Study design: 2011(30), Australia $\begin{aligned} & \text { Cross-sectional, } \\ & \text { quasi-experimental }\end{aligned}$ design

Service type: Family day

Intervention duration: 4 years

Socioeconomic

characteristics:

No. of experimental Intervention: SEIFA $=44.1$

conditions: $1 \quad$ Comparison: SEIFA $=41.0$

Participants:

Intervention: $n 1$ family day care scheme (28 family day care providers)

Control: $n 18$ family day care schemes (223 family day care providers)

Recruitment rate: Not reported

Number of contacts: Unclear

Description of intervention: Romp \& Chomp was a community-based and communitywide programme that sought to change policy, sociocultural and physical aspects of early childhood environments to favour obesity prevention. The intervention activities had a strong focus on community capacity building and developing sustainable changes in areas of policy, sociocultural and physical environments by using a socioecological framework. Project objectives included increasing the capacity of organisations to promote healthy eating and active play, decreasing the consumption of high-sugar drinks, energy-dense snacks and

television viewing time and promoting the consumption of water, milk, fruit and vegetables and increasing active play. Implementation strategies: Educational meetings, educational outreach visits, educational materials and others. Unclear who delivered the intervention - 'a management committee of stakeholder representatives oversaw the implementation of the projects "action" implementation of the projects "action"
plan Community based intervention' plan Community based intervention'
Theoretical underpinning: Guided by the Theoretical underpinning: Guided
health promotion principles of Nutbeam ${ }^{(38,39)}$ and the socio-ecological model of health $(40,41)$

Delivery modalities: Unclear

Description of control: Not reported sample matched to the intervention community's characteristics
Data collection method: The environmental audit used in this evaluation was developed specifically for Romp \& Chomp. The audit comprised forty-five questions designed to capture the general characteristics of the setting (e.g. number of children cared for) and factors in the physical, policy, sociocultural and economic environments of the setting that could enhance or inhibit efforts to promote healthy eating and active play for children aged $0-5$ years who attend the setting.

Validity of measures used: Not reported

Child outcomes: Not reported

Environmental outcomes: Compared with the comparison sample, intervention children spent less time watching television (coefficient -8.01 , $95 \% \mathrm{Cl}-14.90,-1.13, P=0.03)$ and using computer/electronic games (coefficient -1.65 , $95 \% \mathrm{Cl}-3.12,-0.19, P=0.03)$, less time in organised active play (coefficient $-32.52,95 \% \mathrm{Cl}$ $-44.04,-21.01, P<0.001)$ and less time in free inside play (coefficient $-15 \cdot 37,95 \% \mathrm{Cl}$ $-29.40,-1.33, P=0.03$ ). In the intervention service, there were also significantly more rules related to healthy eating (coefficient $-0.42,95 \% \mathrm{Cl}$ $0.20,0.65, P<0.001$ ), more care provider $0.20,0.65, P<0.001)$, more care provider
practices that supported positive meal

experiences for children (coefficient $0.19,95 \% \mathrm{C}$ experiences for children (coefficient $0.19,95 \% \mathrm{Cl}$
$0.07,0.31, P<0.001$ ), fewer unhealthy food/ drink items allowed (coefficient $-0.21,95 \% \mathrm{Cl}$ $-0.41,-0.01, P<0.05)$, higher ratings of resources for both nutrition (coefficient 0.63 , $95 \% \mathrm{Cl} 0.27,1.00, P<0.001)$ and physical activity (coefficient $0.85,95 \%$ Cl $0.55,1.16$ $P<0.001)$ and a higher mean rating for the food-related physical environment (coefficient $0.36,95 \% \mathrm{Cl} 0.14,0.57, P<0.001)$. Intervention costs: Not reported Adverse outcomes: Not reported
Sources of funding: Reported

Potential conflicts of interest: Not

reported 


\section{Nublic Health Nutrition}

Table 1 Continued

\begin{tabular}{|c|c|c|c|c|c|}
\hline $\begin{array}{l}\text { First author, year } \\
\text { published, country }\end{array}$ & Methods & Participants & Intervention & Outcomes & Conflicts of interest \\
\hline $\begin{array}{l}\text { Trost 2011 } 1^{(37)} \\
\text { United States }\end{array}$ & $\begin{array}{l}\text { Study design: Quasi- } \\
\text { experimental design } \\
\text { with replication in } \\
\text { three independent } \\
\text { cohorts of family day } \\
\text { care services } \\
\text { No. of experimental } \\
\text { conditions: } 1\end{array}$ & $\begin{array}{l}\text { Service type: Family day } \\
\text { care services } \\
\text { Socioeconomic } \\
\quad \text { characteristics: Not } \\
\text { reported } \\
\text { Participants: } \\
\text { Intervention: } \\
\text { Baseline } n 196 \\
\quad \text { (pre-assessments) } \\
\text { Final follow-up } n 199 \\
\quad \text { (post-assessments) } \\
\text { Analysed at follow-up } n 196 \\
\text { Control: } n \text { 297 } \\
\text { Recruitment: Not reported } \\
\text { Recruitment rate: Not } \\
\quad \text { reported }\end{array}$ & $\begin{array}{l}\text { Intervention duration: } 12 \text { months } \\
\text { Number of contacts: } 5 \\
\text { Description of intervention: Healthy Kansas } \\
\text { Kids programme - a state-wide initiative } \\
\text { focusing on obesity prevention in early } \\
\text { childhood settings via a train-the-trainer } \\
\text { model. Content included ways to create a } \\
\text { healthy lifestyle through nutrition and } \\
\text { activities, importance of movement for } \\
\text { learning, learning experiences to combine } \\
\text { physical activity, food-related activities } \\
\text { and children's books and including } \\
\text { children in food preparation } \\
\text { Implementation strategies: Educational } \\
\text { meetings, educational outreach visits and } \\
\text { educational materials delivered by } \\
\text { experts in the fields of nutrition and } \\
\text { physical activity } \\
\text { Theoretical underpinning: Not reported } \\
\text { Delivery modalities: Face-to-face } \\
\text { Description of control: Not reported - } \\
\text { representative sample of registered family } \\
\text { day care services operating in the state of } \\
\text { Kansas }\end{array}$ & $\begin{array}{l}\text { Data collection method: Nutrition and Physical } \\
\text { Activity Self-Assessment for Child self- } \\
\text { assessment instrument (NAP SACC-SA). } \\
\text { Validity of measures used: NAP SACC-SA scores } \\
\text { for nutrition and physical activity were calculated } \\
\text { by averaging the responses for the nine nutrition } \\
\text { (thirty-six items, Cronbach's } \alpha 0.76 \text { ) and six } \\
\text { physical activity content areas (nineteen items, } \\
\text { Cronbach's } \alpha 0 \cdot 75) \text {, respectively. } \\
\text { Child outcomes: Not reported } \\
\text { Environmental outcomes: Healthy Kansas Kids } \\
\text { family day cares exhibited significant } \\
\text { improvements in healthy eating } \\
\text { (change between } 6.9 \text { and } 7.1 \% \text { ) and physical } \\
\text { activity scores (change between } 15.4 \text { and } \\
19.2 \% \text { ) }(P<0.05) \text {. Within each cohort, pre- } \\
\text { intervention scores were not significantly } \\
\text { different from the state average, whereas post- } \\
\text { intervention scores were significantly higher than } \\
\text { the state average. } \\
\text { Intervention costs: Not reported } \\
\text { Adverse outcomes: Not reported }\end{array}$ & $\begin{array}{l}\text { Sources of funding: } \\
\text { Reported } \\
\text { Potential conflicts of } \\
\text { interest: Nil } \\
\text { reported }\end{array}$ \\
\hline
\end{tabular}

SEIFA, socioeconomic index for areas. 
This review included two studies reporting on secondary outcomes, both using quasi-experimental designs with parallel control groups. The risk of bias assessment indicated that studies scored mainly 'high' or 'unclear' risk across the range of criteria it was assessed against. Both de Silva-Sanigorski et al.'s ${ }^{(30)}$ community-wide obesity prevention intervention and Trost et al.'s ${ }^{(37)}$ train-the-trainer intervention found statistically significant improvements in family day care providers' implementation of healthy eating and physical activity environments, relative to control providers. As both studies employed strategies including educational meetings, educational outreach visits and educational materials, this suggests that educational interventions targeting provider's knowledge, attitudes and skills may be promising to improve the healthy eating and physical activity environments in the family day care setting. Such findings are supportive of Francis et al. ${ }^{(15)}$ who found that the constructs of Theory of Planned Behaviour (attitudes, subjective norms, perceived behavioural control and intention) may influence family day care providers' ability to create environments supportive of healthy eating and physical activity. Future interventions in this setting, evaluated via randomised controlled trial design, using validated observational measures and measuring child's diet and physical activtiy as well as cost and adverse outcomes are warranted. Further, interventions targeting a broader range of environmental characteristics as identified in the review by Francis, including nutrition and physical activity policies, nutrition feeding practices, communication with parents, availability of play equipment and creative play spaces, may be warranted to increase the overall impact of interventions on child's diet, physical activity and/or weight status ${ }^{(15)}$.

Despite a clear need for more research in the area, researchers have identified several unique challenges with undertaking intervention research in the family day care setting. Specifically, this includes large variability in the operations and stability of the setting, the small number of clusters (i.e. each provider may only enrol up to ten children), participation burden in a single-carer environment and increased risk of loss to follow-up of family day care schemes, providers and children ${ }^{(22)}$. Further, the personal nature of a relationship between family day care providers and the families of children attending care compared to centre-based care could present additional challenges to implementing obesity prevention initiatives ${ }^{(46)}$. A number of strategies have been suggested to facilitate the conduct of research in this setting, including ensuring recruitment materials are understandable and address barriers to research participation such as lack of time, gaining endorsement from community partners, developing rapport with family day care services; ensuring concerns about participation are addressed; and considering strategies to facilitate parent engagement without jeopardising personal relationships between carers and families ${ }^{(22)}$. 
Although few interventions were examined, findings of this review are important as it highlights gaps in the evidence base and presents clear opportunities for future research in this setting. This review has found no investigations examining the impact of family day care-based interventions on child nutrition, physical activity and weight status measures, and as such, the effectiveness of such interventions on these outcomes is unknown. Additionally, this review provides a high-quality synthesis of the existing evidence base and highlights ongoing research in the area. Such information is valuable to undertake future interventions in this setting. Given the broad eligibility criteria and rigorous search processes, this review presents an accurate overview of the effectiveness of obesity prevention strategies in family day care settings at the time the search was run (March 2019). A future update of this review is needed given the increasing interest in this setting as an avenue to support obesity prevention initiatives and ongoing trials ${ }^{(15,44,45)}$.

\section{Strengths and limitations}

This review applied high-quality processes in line with those recommended by the Cochrane Collaboration ${ }^{(24)}$. Given the limited number of studies and heterogeneous outcomes, we were unable to undertake a meta-analyses of study outcomes. Further, the risk of bias for the included studies was judged as 'high' or 'unclear' for a number of criteria, and findings from this review should be considered in light of such assessments. Non-English studies were excluded from the review, and no systematic search of grey literature was undertaken, which could have resulted in missed studies.

\section{Conclusion}

This review has found no controlled trial examining the impact of interventions on child's diet, physical activity and/or weight status in family day care services. However, two quasi-experimental obesity prevention interventions were found to improve family day care's healthy eating and physical activity environments. These findings highlight a clear need for future randomised controlled trials measuring the impact on both child outcomes and family day care environments in this setting.

\section{Acknowledgements}

Acknowledgements: The authors wish to acknowledge Debbie Booth for her assistance with developing the search strategy, and Luke Wolfenden for providing feedback on the draft manuscript. Financial support: This study received systematic review pilot funding from the Priority Research Centre for Health Behaviour, University of
Newcastle. Hunter New England Population Health and the University of Newcastle provided infrastructure funding. S.L.Y. is supported by an Australian Research Council Discovery Early Career Researcher Award (DE170100382). Conflict of interest: None. Authorship: S.L.Y., J.J. and M.F. developed the review question. S.L.Y., M.L. and L.K.C. developed the search strategy. S.L.Y., M.L., A.G., E.K., M.F., S.M., L.K.C. and K.S. screened the articles. A.G. and M.L. conducted data extraction and risk of bias assessment. S.L.Y. led the drafting of the manuscript, supported by M.L. and T.D. All authors were involved in reviewing the draft manuscript, supporting interpretation of results and final approval of the submitted versions. Ethics of buman subject participation: Not applicable.

\section{Supplementary material}

For supplementary material accompanying this article visit https://doi.org/10.1017/S1368980019005275

\section{References}

1. World Health Organisation (2018) Childhood Overweight and Obesity. World Health Organisation. http://www.who. int/dietphysicalactivity/childhood/en/ (accessed May 2020).

2. Reilly JJ \& Kelly J (2011) Long-term impact of overweight and obesity in childhood and adolescence on morbidity and premature mortality in adulthood: systematic review. Int $J$ Obes 35, 891.

3. Reilly JJ, Methven E, McDowell ZC et al. (2003) Health consequences of obesity. Arch Dis Child 88, 748-752.

4. Umer A, Kelley GA, Cottrell LE et al. (2017) Childhood obesity and adult cardiovascular disease risk factors: a systematic review with meta-analysis. BMC Public Health 17, 683 .

5. World Health Organization (2018) Fact Sheet: Obesity and Overweight. Geneva: World Health Organization.

6. Hinkley T, Salmon J, Okely AD et al. (2012) Preschoolers' physical activity, screen time, and compliance with recommendations. Med Sci Sports Exerc 44, 458-465.

7. Kranz S, Findeis JL \& Shrestha SS (2008) Use of the revised children's diet quality index to assess preschooler's diet quality, its sociodemographic predictors, and its association with body weight status. J Pediatr 84, 26-34.

8. World Health Organization (2012) Population-Based Approaches to Childhood Obesity Prevention. Geneva: World Health Organization.

9. Australian Government (2018) Early Childhood Education and Care in Summary Report: June Quarter 2018. In Training DoEa, editor.

10. Australian Government (2019) Child Care in Australia. In Training DoEa, editor.

11. Department of Education and Training (2018) Child Care Provider Handbook. In Training DoEa, editor. Canberra: Australian Government.

12. Laughlin L (2013) Who's Minding the Kids? Child Care Arrangements: Spring 2011. In Commerce USDo, editor.

13. Childcare Aware (2015) Parents and the High Cost of Child Care, Arlington, Virginia. 
14. Benjamin-Neelon SE, Vaughn AE, Tovar A et al. (2018) The family child care home environment and children's diet quality. Appetite 126, 108-113.

15. Francis L, Shodeinde L, Black MM et al. (2018) Examining the obesogenic attributes of the family child care home environment: a literature review. J Obes 2018, 20.

16. National Association for Sport and Physical Education (2002) Active Start: A Statement of Physical Activity Guidelines for Children Birth to Five Years. Reston, VA: National Association for Sport and Physical Education.

17. Vanderloo LM (2014) Screen-viewing among preschoolers in childcare: a systematic review. BMC Pediatr 14, 205.

18. Tovar A, Benjamin-Neelon SE, Vaughn AE et al. (2018) Nutritional quality of meals and snacks served and consumed in family child care. J Acad Nutr Diet 118, 2280-2286.

19. Temple M \& Robinson JC (2014) A systematic review of interventions to promote physical activity in the preschool setting. J Spec Pediatr Nurs 19, 274-284.

20. Mikkelsen MV, Husby S, Skov LR et al. (2014) A systematic review of types of healthy eating interventions in preschools. Nutr J 13, 56.

21. Wolfenden L, Jones J, Finch M et al. (2016) Strategies to improve the implementation of healthy eating, physical activity and obesity prevention policies, practices or programmes within childcare services. Cochrane Database Syst Rev issue 10, CD011779.

22. Ward DS, Vaughn AE, Burney RV et al. (2016) Recruitment of family child care homes for an obesity prevention intervention study. Contemp Clin Trials Commun 3, 131-138.

23. Tovar A, Vaughn AE, Grummon A et al. (2017) Family child care home providers as role models for children: cause for concern? Prev Med Rep 5, 308-313.

24. Higgins J \& Green S (2019) Cochrane Handbook for Systematic Reviews of Interventions version 5.1.0 (updated March 2011). The Cochrane Collaboration. Chichester (UK): John Wiley \& Sons.

25. Moher D, Liberati A, Tetzlaff J et al. (2009) Preferred reporting items for systematic reviews and meta-analyses: the PRISMA statement. BMJ 339, b2535.

26. Veritas Health Innovation Covidence Systematic Review Software. Melbourne, Australia.

27. The Cochrane Public Health Group (2011) Guide for Developing a Cochrane Protocol. Melbourne, Australia. https://ph.cochrane.org/sites/ph.cochrane.org/files/public/ uploads/Guide\%20for\%20PH\%20protocol_Nov\%202011_ final\%20for $\% 20$ website.pdf (accessed May 2020).

28. Effective Practice and Organisation of Care (EPOC) (2015) Epoc Taxonomy. epoc.cochrane.org/epoc-taxonomy (accessed May 2020).

29. Bravo A, Cass Y \& Tranter D (2008) Good food in family day care: improving nutrition and food safety in family day care. Nutr Diet 65, 47-55.

30. de Silva-Sanigorski A, Elea D, Bell C et al. (2011) Obesity prevention in the family day care setting: impact of the romp \& chomp intervention on opportunities for children's physical activity and healthy eating. Child Care Health Dev 37, 385-393.
31. Dev DA, Williams N, Iruka I et al. (2018) Improving the nutrition and screen time environment through selfassessment in family childcare homes in Nebraska. Public Health Nutr 21, 2351-2359.

32. Dinkel D, Dev D, Guo Y et al. (2018) Improving the physical activity and outdoor play environment of family child care homes in Nebraska through go nutrition and physical activity self-assessment for child care. $J$ Phys Act Health 15, 730-736.

33. Naylor PJ \& Temple VA (2013) Enhancing the capacity to facilitate physical activity in home-based child care settings. Health Promot Pract 14, 30-37.

34. Trost SG, Messner L, Fitzgerald K et al. (2009) Nutrition and physical activity policies and practices in family child care homes. Am J Prev Med 37, 537-540.

35. Kao J, Woodward-Lopez G, Kuo ES et al. (2018) Improvements in physical activity opportunities: results from a community-based family child care intervention. Am J Prev Med 54, Suppl. 2, S178-S185.

36. Woodward-Lopez G, Kao J, Kuo ES et al. (2018) Changes in nutrition policies and dietary intake in child care homes participating in healthy eating and active living initiative. Am J Prev Med 54, Suppl. 2, S170-S177.

37. Trost SG, Messner L, Fitzgerald K et al. (2011) A nutrition and physical activity intervention for family child care homes. Am J Prev Med 41, 392-398.

38. Nutbeam D (1999) Evaluating health promotion. BMJ 318 404A.

39. Nutbeam D (1998) Evaluating health promotion - progress, problems and solutions. Health Promot Int 13, 27-44.

40. McLeroy KR, Bibeau D, Steckler A et al. (1988) An ecological perspective on health promotion programs. Health Educ $Q$ 15, 351-377.

41. Swinburn B, Egger G \& Raza F (1999) Dissecting obesogenic environments: the development and application of a framework for identifying and prioritizing environmental interventions for obesity. Prev Med 29, 563-570.

42. Benjamin SE, Ammerman A, Sommers J et al. (2007) Nutrition and Physical Activity Self-Assessment for Child Care (NAP SACC): results from a pilot intervention. J Nutr Educ Behav 39, 142-149.

43. Benjamin SE, Neelon B, Ball SC et al. (2007) Reliability and validity of a nutrition and physical activity environmental self-assessment for child care. Int J Behav Nutr Phys Act 4, 29.

44. Ostbye T, Mann CM, Vaughn AE et al. (2015) The keys to healthy family child care homes intervention: study design and rationale. Contemp Clin Trials 40, 81-89.

45. Risica PM, Tovar A, Palomo V et al. (2019) Improving nutrition and physical activity environments of family child care homes: the rationale, design and study protocol of the 'healthy start/comienzos sanos' cluster randomized trial. BMC Public Health 19, 419.

46. Bromer J (2001) Helpers, mothers, and preachers: the multiple roles and discourses of family child care providers in an African-American community. Early Child Res $Q$ 16, 313-327. 